\title{
Glutamine and norepinephrine in follicular fluid synergistically affect the antioxidant capacity of human granulosa cells and the outcome of IVF-ET
}

\section{Lulu Wang}

The International Peace Maternity and Child Health Hospital, Shanghai Jiao Tong University

Chengliang Zhou

The International Peace Maternity and Child Health Hospital, Shanghai Jiao Tong University

Junyan Sun

The International Peace Maternity and Child Health Hospital, Shanghai Jiao Tong University

Qiuwan Zhang

The International Peace Maternity and Child Health Hospital, Shanghai Jiao Tong University

Dongmei Lai ( $\nabla$ laidongmei@hotmail.com )

The International Peace Maternity and Child Health Hospital, Shanghai Jiao Tong University

\section{Research Article}

Keywords: Neurotransmitter metabolism, Follicular fluid, Granulosa cells, Glutamine, Norepinephrine, IDH1 gene

Posted Date: February 23rd, 2022

DOI: https://doi.org/10.21203/rs.3.rs-1255886/v2

License: (c) (1) This work is licensed under a Creative Commons Attribution 4.0 International License. Read Full License 


\section{Abstract}

Background: An increasing number of studies have demonstrated that changes in metabolic levels in follicular fluid are directly related to oocyte maturation, fertilization, the quality of embryo and pregnancy rates. However, whether the intra-follicular neurotransmitters affect the function of granulosa cells and the outcome of in vitro fertilization and embryo transfer (IVF-ET) is not clear.

Methods: Human follicular fluid and cumulus granulosa cells (GCs) were harvested from large follicles obtained from 27 patients undergoing IVF. Neurotransmitters and steroid hormones in follicular fluid were measured through liquid chromatography-tandem mass spectrometry (LC-MS/MS) and highperformance liquid chromatography-mass spectrometry (HPLC-MS/MS) respectively. Based on the content of Glutamine (GIn) in follicular fluid, the samples were divided into two groups: high Gln level group and low GIn level group. The expression of proliferation-, steroidogenesis- and antioxidant-related genes in GCs was detected by qRT-PCR. In vitro experiments, KGN cells were cultured with or without GIn and NE followed by western blotting to detect PCNA and IDH1 expression. EdU staining and ELISA were conducted to evaluate GCs proliferation and measure the contents of 17 b-estradiol (E2) and progesterone (Prog) in the conditioned medium. Primary and secondary outcomes were the number of mature and retrieved oocytes, and the ratio of high quality embryos, respectively.

Results: Gln and norepinephrine (NE) were abundant neurotransmitters in follicular fluid. The contents of GIn and NE in follicular fluid exhibited a significantly positive correlation. The contents of GIn and NE in follicular fluid affected the function of granulosa cells, including their proliferative potential, E2 production and antioxidant capacity. Moreover, the concentrations of GIn and NE in follicular fluid showed significantly positive correlation with isocitrate dehydrogenase 1 (IDH1) expression in GCs. Importantly, the increased GIn and NE in follicular fluid partially affected the quality of embryonic development by regulating IDH1 expression in GCs. In vitro studies further demonstrated that GIn and NE played synergistically function in improving GCs proliferation and increasing the E2 production by upregulating IDH1 expression.

Conclusions: These data demonstrate that neurotransmitter metabolic levels in follicular fluid affect the function of cumulus GCs. As important neurotransmitter metabolites, Gln and NE play significant roles in promoting GCs proliferation and increasing E2 production in granulosa cells. In addition, intrafollicular GIn and NE could upregulate the expression of the antioxidative gene IDH1 in cumulus GCs, which could be a good predictor for selecting optimal quality oocytes and evaluating the quality of embryonic development.

\section{Background}

Subfertility, also commonly referred to infertility, is a reproductive system disease characterized by failure to establish a clinical pregnancy after normal unprotected sexual intercourse for 12 months or more, or impairment of the reproductive capacity of an individual or with his or her partner $[1,2]$. Subfertility has 
been recognized as a worldwide public health problem by the World Health Organization (WHO), and approximately half of these cases lack an definitive explanation for pregnancy failure [3]. It is estimated that in western countries, one in six couples has subfertility, which is recognized as the cause of psychological distress in both men and women [4]. Assisted reproductive technology (ART) including in vitro fertilization (IVF) and intracytoplasmic sperm injection (ICSI) is a promising option with suitable interventions for the clinical management of subfertility [5]. IVF offers a possibility for achieving pregnancy, but the success rate is still suboptimal $[6,7]$.

A healthy intrafollicular environment supports the production of developing oocytes and affects oocyte quality by coordinating the cumulus GCs and the follicular fluid [8]. Follicular fluid originates from both plasma and secretion by cumulus GCs. As an important biofluid, follicular fluid provides a special microenvironment for follicular development and impacts oocyte quality, implantation and early embryo development. Follicular fluid is rich in proteins, including sex hormones, nutrient substances, cytokines, growth factors and neurotransmitters, which is critical for oocyte development [9]. Cumulus granulosa cells represent the predominant somatic cell type of follicle development and are involved in steroidogenesis and folliculogenesis. Furthermore, they connect with oocytes via direct gap junctions to support the developing oocyte [10-12]. The process of follicular development involves local biochemical exchanges and substantial modifications in cellular metabolism.

Previous studies have demonstrated that NE in the follicular fluid derived from the sympathetic neurons of the peripheral nervous system in the mammalian ovary takes part in the regulation of ovarian function $[13,14]$. Follicular cells such as granulosa and theca express $\beta$-adrenergic receptor in respond to the $\beta$ adrenergic agonist, leading to a physiological follicular development, ovulation and the synthesis and production of steroid hormones [15]. In addition, NE and its metabolites could induce the generation of reactive oxygen species (ROS) in human GCs [16]. Once the level of NE concentration exceeds the physiological concentration, it may lead to ROS-related pathophysiology events including oxidative stress and cell death [17]. Thus, the content of NE in the follicular microenvironment could be a key physiology or physiological indicator for the signaling in steroidogenic Leydig cells and the process of ovulation.

Amino acids serve as substrates for the synthesis of proteins, not only enhancing embryonic development but also affecting blastocyst formation in mice. A study demonstrated that Glycine (Gly), Glutamine (Gln), alanine (Ala), Glutamate (Glu) and Proline (Pro) were the most abundant amino acids in follicular fluid [18]. The disturbance of follicular amino acid metabolism is related to women with polycystic ovary syndrome (PCOS), which may be the reason for the poor pregnancy outcome in obese patients and increased risk of abortion in PCOS patients [19]. In addition, they are not only components of proteins, but also regulate neurotransmitter homeostasis, such as a metabolite shuttle known as the glutamate/GABA-glutamine cycle describes the release of neurotransmitter glutamate or GABA from neurons and subsequent uptake into astrocytes, play import roles in nervous system [20,21]. However, whether the neurotransmitters metabolic levels provided by follicular fluid influences the function and hormone synthesis of cumulus granulosa cells need to be further investigated. 
Oxidative stress plays an important role in reproductive processes, including follicular development, oocyte maturation, ovulation and fertilization, as well as embryo implantation and embryo development [22]. Excessive generation of ROS, if not efficiently counterbalanced by antioxidative enzymes, may cause damage to oocytes and granulosa cells, leading to poor oocyte quality [11]. In addition, increased levels of endogenous ROS and reduced antioxidant genes expression diminish cellular antioxidant defense capability, resulting in oxidative damage to lipids, proteins and DNA in ovary [23]. In our previous study, psychological stress-induced oxidative stress decreased the expression of the antioxidant gene IDH1 to disturb and compromise the function of granulosa cells, accelerating ovarian senescence [24]. Antioxidant treatment with melatonin can provide reproductive medicine with a way to improve IVF outcomes [25]. Additionally, antioxidant supplementation in IVF culture medium could increase fertilization rates and subsequent healthy embryo development [26]. However, little is known about the impact of neurotransmitters in follicular fluid on oxidative capacity of granulosa cells.

During the process of follicle growth to ovulation, which is mediated by the preovulation production of gonadotropins, metabolic profiles change dynamically in follicular fluid. Thus, a better understanding of the metabolic levels in follicular fluid is important for exploring the metabolic cooperativity between oocytes and cumulus granulosa cells. In recent years, metabolomics, as a new approach, has been used to explore various molecular mechanisms involved in tissues and organs. Among the various metabolomics techniques, LC-MS/MS is widely used as a conventional detection method for analyzing small molecules, which could provide a quantitative evaluation with minimal handling [27].

In this study, we identified the neurotransmitters in follicular fluid from IVF patients by high sensitivity, convenient and fast LC-MS/MS technology and evaluated the impact of neurotransmitter GIn and NE in follicular fluid on the function and antioxidative capacity of cumulus granulosa cells, which could impact the clinical outcome of IVF patients. Additional in vitro cell experiments were performed to explore the effects of GIn and NE on the proliferation and steroidogenesis of granulosa cells.

\section{Methods}

\section{Study design}

This is an observational study of infertile women before and during IVF treatment at the International Peace Maternity and Child Health Hospital (IPMCH), Shanghai, China. Follicular fluid and cumulus granulosa cells on the day of oocyte retrieval were obtained from 27 patients undergoing IVF at the reproductive center. The subjects were recruited with male factor infertility or tubal factor infertility. Subjects with endometriosis and PCOS were excluded. The age of the patients ranged from 25 to 40 years, and the size of the follicles ranged from 19 to $24 \mathrm{~mm}$.

\section{Collection of GCs and follicular fluid}

The size of the follicle was estimated by ultrasound at the time of oocyte retrieval. Follicular aspirates contain oocytes surrounded by cumulus GCs. After the oocytes were removed by the embryologist, the 
remaining material was centrifuged at $3000 \mathrm{~g}$ for 15 mins to isolate the GCs. The fluid from the first aspirated follicle was used to measure hormone and neurotransmitter metabolic levels.

\section{Quantification of neurotransmitters in follicular fluid by liquid chromatography-tandem mass spectrometry (LC-MS/MS)}

Human follicular fluid was prepared for neurotransmitters metabolomics detection. Twenty-three metabolites (Ach: Acetylcholine chloride; Gln: Glutamine; Glu: Glutamate; His: L-Histidine; NE: norepinephrine; Tyr: Tyrosine; Trp: Tryptophan; Kyn: Kynurenine; GABA: 4-Aminobutyric acid; HisA: Histamine; PA: Picolinic acid; TyrA: Tyramine; DA: Hydroxytyramine hydrochloride; TrpA: Tryptamine; 5-HT: Serotonin hydrochloride; E: Adrenaline hydrochloride; KynA: Kynurenic acid; 5-HIAA: 5-Hydroxyindole-3acetic acid; DOPA: Levodopa; XA: Xanthurenic acid; VWA: Vanillymandelic Acid; 5-HTTP: 5Hydroxytryptophan; MT: Melatonine) were simultaneously reported in LC-MS/MS as described previously[28, 29]. Data analysis was performed with Analyst 1.6 software.

\section{RNA Extraction and qRT-PCR}

Granulosa cells were collected in TRIzol reagent (Invitrogen, Carlsbad, CA, USA), and total RNA was extracted according to previously described methods[24]. A total of 1 mg RNA was converted to cDNA with a Takara kit (Applied Biosystems Foster City, CA, USA; Takara, Shiga, Japan). The genes of interest were amplified with a 7900HT fast real-time PCR system (Applied Biosystems) and SYBR Green Real-time PCR Master Mix (Applied Biosystems/Takara). The PCR primers were designed according to the cDNA sequences in the NCBI database. The primer sequences used are shown in Table 1. The cycling conditions used for the PCR analysis were as follows: $95^{\circ} \mathrm{C}$ for $5 \mathrm{~s}, 60^{\circ} \mathrm{C}$ for $30 \mathrm{~s}$, and $72^{\circ} \mathrm{C}$ for $30 \mathrm{~s}(40$ cycles). $18 \mathrm{~S}$ was used as an internal control. The $2^{-\Delta \Delta C t}$ method was employed to determine the relative mRNA expression level. 
Table 1

Primer sequences for qRT-PCR in human granulosa cells

\begin{tabular}{|c|c|c|}
\hline Target genes & Primer sequence(5'-3') & Product Size (bp) \\
\hline \multirow[t]{2}{*}{ IDH1 } & F: AGAAGCATAATGTTGGCGTCA & $106 \mathrm{bp}$ \\
\hline & R: CGTATGGTGCCATTTGGTGATT & \\
\hline \multirow[t]{2}{*}{ GPX1 } & F: CCGGGACTACACCCAGATGA & $101 \mathrm{bp}$ \\
\hline & R: CGTTCTCCTGATGCCCAAAC & \\
\hline \multirow[t]{2}{*}{ NRF2 } & F: TCAGCGACGGAAAGAGTATGA & $174 \mathrm{bp}$ \\
\hline & R: CCACTGGTTTCTGACTGGATGT & \\
\hline \multirow[t]{2}{*}{ KEAP1 } & F: CTGGAGGATCATACCAAGCAGG & $220 \mathrm{bp}$ \\
\hline & R: GGATACCCTCAATGGACACCAC & \\
\hline \multirow[t]{2}{*}{ PCNA } & F: CCTGCTGGGATATTAGCTCCA & $109 \mathrm{bp}$ \\
\hline & R: CAGCGGTAGGTGTCGAAGC & \\
\hline \multirow[t]{2}{*}{ CCND2 } & F: TTTGCCATGTACCCACCGTC & $104 \mathrm{bp}$ \\
\hline & R: AGGGCATCACAAGTGAGCG & \\
\hline \multirow[t]{2}{*}{ LHR } & F: CACATAACCACCATACCAGGAAA & $124 / 283 b p$ \\
\hline & R: AAGTCAGTGTCGTCCCATTGA & \\
\hline \multirow[t]{2}{*}{ FSHR } & F: AAAGCTGCCTACTCTGGAAAAG & $170 \mathrm{bp}$ \\
\hline & R: GACCCCTAGCCTGAGTCATATAA & \\
\hline \multirow[t]{2}{*}{ STAR } & F: GTGGAACCCCAGTGTCAAGA & $129 \mathrm{bp}$ \\
\hline & R: CAGCGCACACTCACAAAGTC & \\
\hline \multirow[t]{2}{*}{ CYP11A } & F: GAGGCCCAGCGATTCATTGAT & $94 \mathrm{bp}$ \\
\hline & R: TCCTGAACAGACGGAACAGGT & \\
\hline \multirow[t]{2}{*}{ CYP19A } & F: TGGAAATGCTGAACCCGATAC & $161 \mathrm{bp}$ \\
\hline & R: AATTCCCATGCAGTAGCCAGG & \\
\hline \multirow[t]{2}{*}{ HSD-3 $\beta$} & F: CTTGTGCGTTAAGACCCACAT & $124 \mathrm{bp}$ \\
\hline & R: GGGTTGACTGTAGAGAACTTTCC & \\
\hline \multirow[t]{2}{*}{ HSD-17 $\beta$} & F: GTGCTGGTGTGTAACGCAG & $98 \mathrm{bp}$ \\
\hline & R: GTCCCTACTACATTCACGTCCA & \\
\hline 18sRNA & F: GTAACCCGTTGAACCCCATT & $151 \mathrm{bp}$ \\
\hline
\end{tabular}




\section{Target genes Primer sequence(5'-3') Product Size (bp)}

R: CCATCCAATCGGTAGTAGCG

\section{Hormone metabolic levels in the follicular fluid}

The concentrations of twenty kinds of hormones in the follicular fluid were detected by high-performance liquid chromatography-mass spectrometry (HPLC-MS/MS) as described previously[30]. In addition, the concentration of testosterone and cortisol in the follicular fluid was detected by the chemiluminescence method[31].

\section{Human Granulosa Tumor Cell Line (KGN) culture and treatment}

The human ovarian granulosa cell line was kindly gifted by Dr. Zuwei Yang (IPMCH, School of Medicine, Shanghai Jiao Tong University, Shanghai, China). KGN was initially cultured in Dulbecco minimal essential medium (DMEM)-F12 medium (Gibco, Grand Island, NY, USA) supplemented with $10 \%$ fetal calf serum and antibiotics (100 IU/mL penicillin, $100 \mu \mathrm{g} / \mathrm{mL}$ streptomycin) in a $5 \% \mathrm{CO}_{2}$ atmosphere at $37^{\circ} \mathrm{C}$. After deprivation of glutamine for $24 \mathrm{~h}$, the cells were treated with the corresponding conditioned medium for $48 \mathrm{~h}$.

\section{Enzyme-linked immunosorbent assay (ELISA)}

Cell culture supernatant samples were collected and centrifuged for $20 \mathrm{~min}$ at $2500 \mathrm{rpm}$ to acquire the supernatant at $-4^{\circ} \mathrm{C}$. The supernatant was stored at $-80^{\circ} \mathrm{C}$ for further analysis. The concentration of $17 \beta-$ estradiol and progesterone in cell culture supernatant was measured by ELISA kits (Xinle Biological Technology, Shanghai, China), according to the manufacturer's instruction.

\section{Western blotting}

Proteins were extracted from cultured cells with RIPA lysis buffer (Yeasen, Shanghai, China) and protein concentration was measured via a BCA kit (Thermo Scientific, Rockford, IL, USA). The protocol for western blotting is reported previously[24]. The following primary antibodies were used: rabbit antibodies against $\beta$-tubulin (1:1000, CST, MA, USA) and anti-PCNA antibody (1:1000, CST, MA, USA). After incubation with primary antibodies at $4^{\circ} \mathrm{C}$ overnight, the membranes were incubated with horseradish peroxidaseconjugated secondary antibodies (1:2500, CST, MA, USA) for 1 hour at room temperature. The bands were detected by electrochemiluminescence (ECL, Millipore, Billerica, MA, USA). The relative intensity of the target proteins was normalized to $\beta$-tubulin using ImageJ software ( $\mathrm{NIH}$, Bethesda, MD, USA).

\section{EdU Staining}

The proliferation of KGN cell was evaluated by EdU staining (RiboBio, Guangdong, China). Briefly, EdU was added to the cell culture medium at a concentration of $10 \mu \mathrm{M}$. Cells were cultured for 24 hours and stained with Alexa 594 and DAPI. Images were captured under a microscope (Leica, WetzlaR, Germany) 
and positive fluorescence signals were counted. The number of EdU-positive cells per 100 cells was evaluated.

\section{Statistical analysis}

All statistical analyses were performed using Prism software, and a $p$ value of $<0.05$ was considered statistically significant. Means and standard error were used as descriptive statistics. T-tests were used to compare different variables between the low-level and high-level glutamine groups. Linear correlation analysis was used to compare the expression of the IDH1 gene and steroidogenesis in luteinized granulosa cells. In the current study, when a power analysis was performed with $80 \%$ power and an a value of 0.05 , the number of samples in each group needed to be 13 to confirm statistical significance.

\section{Ethics approval and informed consent}

Informed consents for participating in this study were obtained from all the patients and this study was approved by the Institutional Ethics Committee of the International Peace Maternity and Child Health Hospital. All experiments were performed in accordance with the relevant guidelines and regulations.

\section{Results}

\section{Glutamine is positively correlated with norepinephrine content in the follicular fluid of IVF patients}

Dynamic metabolic changes within the follicular fluid are important for cumulus expansion, which represents the consequence of oocyte cumulus complex (OCC) metabolic cooperation. In this study, we first measured the content of neurotransmitters in follicular fluid by LC-MS/MS. The results regarding the concentrations of Ach, Gln, Glu, His, NE, Tyr, Trp, Kyn in human follicular fluid are shown in Table 2. The other fifteen neurotransmitters were not detected in most of the follicular fluid samples. Correlation analysis showed that GIn was positively correlated with the NE content in follicular fluid $(R=0.5869$, $P<0.005$, Figure 1A). However, there was no relationship between the contents of Gln and Glu in follicular fluid $(R=0.2374, P>0.05$, Figure 1B). 
Table 2

Concentration of multiple neurotransmitters in human follicular fluid

\begin{tabular}{|c|c|c|c|c|c|c|c|c|}
\hline \multirow[t]{2}{*}{ Sample ID } & Ach & Gln & Glu & His & NE & Tyr & Trp & Kyn \\
\hline & (ug/ml) & (ug/ml) & (ug/ml) & (ug/ml) & (ug/ml) & (ug/ml) & (ug/ml) & (ug/ml) \\
\hline 01 & 0.019 & 45.715 & 21.989 & 1.083 & 0.233 & 6.431 & 7.227 & 0.238 \\
\hline 02 & 0.025 & 49.37 & 10.045 & 1.472 & 0.281 & 6.231 & 7.373 & 0.548 \\
\hline 03 & 0.080 & 60.028 & 16.639 & 3.620 & 0.401 & 19.822 & 5.658 & 0.757 \\
\hline 04 & 0.018 & 49.313 & 15.104 & 1.365 & 0.251 & 4.751 & 6.977 & 0.307 \\
\hline 05 & 0.019 & 52.869 & 16.819 & 1.510 & 0.240 & 5.724 & 7.711 & 0.350 \\
\hline 06 & 0.016 & 54.879 & 13.450 & 1.273 & 0.245 & 6.297 & 5.830 & 0.202 \\
\hline 07 & 0.019 & 35.712 & 17.795 & 1.074 & 0.253 & 5.903 & 6.359 & 0.361 \\
\hline 08 & 0.027 & 44.439 & 11.364 & 1.389 & 0.244 & 6.018 & 4.618 & 0.307 \\
\hline 09 & 0.018 & 51.359 & 12.867 & 1.329 & 0.258 & 6.633 & 6.925 & 0.319 \\
\hline 10 & 0.023 & 60.335 & 15.089 & 0.947 & 0.214 & 7.698 & 6.458 & 0.243 \\
\hline 11 & 0.020 & 44.826 & 13.077 & 1.046 & 0.202 & 4.362 & 5.838 & 0.284 \\
\hline 12 & 0.026 & 42.962 & 14.758 & 1.339 & 0.130 & 5.837 & 5.290 & 0.272 \\
\hline 13 & 0.021 & 58.259 & 13.474 & 1.411 & 0.235 & 5.413 & 5.804 & 0.225 \\
\hline 14 & 0.017 & 56.594 & 16.155 & 1.208 & 0.285 & 7.238 & 10.141 & 0.241 \\
\hline 15 & 0.02 & 52.631 & 6.706 & 1.455 & 0.142 & 10.169 & 9.157 & 0.255 \\
\hline 16 & 0.018 & 44.161 & 10.595 & 1.417 & 0.144 & 5.729 & 6.591 & 0.455 \\
\hline 17 & 0.017 & 37.091 & 14.466 & 1.146 & 0.231 & 4.984 & 7.835 & 0.346 \\
\hline 18 & 0.021 & 33.855 & 8.973 & 1.014 & 0.143 & 4.525 & 5.623 & 0.261 \\
\hline 19 & 0.016 & 50.998 & 10.091 & 1.029 & 0.180 & 6.335 & 8.394 & 0.243 \\
\hline 20 & 0.014 & 44.261 & 11.385 & 1.012 & 0.186 & 4.101 & 6.881 & 0.284 \\
\hline 21 & 0.018 & 50.524 & 11.722 & 1.249 & 0.205 & 6.630 & 5.096 & 0.199 \\
\hline 22 & 0.018 & 38.522 & 11.873 & 1.055 & 0.096 & 5.849 & 4.797 & 0.265 \\
\hline 23 & 0.017 & 37.578 & 10.232 & 1.099 & 0.113 & 4.864 & 5.351 & 0.248 \\
\hline 24 & 0.018 & 42.237 & 14.710 & 1.106 & 0.158 & 6.031 & 5.536 & 0.323 \\
\hline 25 & 0.027 & 44.286 & 11.252 & 1.313 & 0.109 & 5.851 & 6.290 & 0.262 \\
\hline 26 & 0.022 & 45.651 & 11.975 & 1.157 & 0.218 & 7.406 & 7.377 & 0.336 \\
\hline
\end{tabular}




\begin{tabular}{|c|c|c|c|c|c|c|c|c|}
\hline \multirow[t]{2}{*}{ Sample ID } & Ach & Gln & Glu & His & NE & Tyr & Trp & Kyn \\
\hline & (ug/ml) & $(\mathrm{ug} / \mathrm{ml})$ & (ug/ml) & (ug/ml) & $(\mathrm{ug} / \mathrm{ml})$ & $(\mathrm{ug} / \mathrm{ml})$ & (ug/ml) & (ug/ml) \\
\hline 27 & 0.026 & 33.927 & 8.471 & 1.103 & 0.127 & 5.956 & 5.352 & 0.348 \\
\hline
\end{tabular}

Gln serves as a substrate for the synthesis of proteins in follicular fluid, which directly promotes oocyte nuclear maturation and enhances embryo development. However, the effect of GIn and NE in follicular fluid on the function of cumulus GCs is still unclear. To further investigate the function of GIn and NE in follicular fluid, 27 samples were divided into two groups according to their concentration of Gln, the high Gln level group $(>45 \mu \mathrm{g} / \mathrm{mL})$ and low Gln level group $(<45 \mu \mathrm{g} / \mathrm{mL})$.

\section{Effect of intra-follicular glutamine/norepinephrine on the function of granulosa cells}

The function of GCs involved in a proliferative capacity, receptor expression and steroid hormone synthesis in cumulus GCs was determined by qRT-PCR. Compared with the low Gln level group, those with high Gln level had a significant 2-fold increase in proliferating cell nuclear antigen (PCNA) and cyclin D2 (CCND2) mRNA levels in the GCs $(P<0.001$ and $P<0.01$, Figure $2 A-B)$. However, there were no statistically significant differences in luteinizing hormone receptor $(L H R)$ or follicle stimulating hormone receptor (FSHR) mRNA levels between the two groups $(P>0.05$, Figure 2C-D). Notably, the mRNA levels of the steroid hormone synthesis-related genes steroid acute regulatory protein (STAR), cytochrome P450 (CPY11A, CYP19A) and hydroxysteroid dehydrogenase-3beta (HSD-3B) significantly decreased in the low Gin level group $(P<0.05$, Figure $2 E-H)$. However, there was no significant difference in hydroxysteroid dehydrogenase-17beta $(H S D-17 \beta)$ mRNA levels between the two groups $(P>0.05$, Figure 21$)$. These data demonstrated that the increased GIn and NE in the follicular fluid could promote the proliferation and steroidogenesis of GCs.

\section{Glutamine/norepinephrine promote estrogen synthesis in the follicular fluid of IVF patients}

To obtain an overview of hormone metabolic characteristics in follicular fluid between high and low GIn level groups, targeted metabolic profiling was conducted using HPLC-MS/MS. Nine kinds of hormones were detected in the two groups, including estrone, estriol, 17 $\beta$-estradiol, progesterone, methyl testosterone, (D)-norgestrel, 19-nortestosterone, epitestosterone and medroxy progesterone. In addition, the concentration of testosterone in follicular fluid was measured by a chemiluminescence immunoassay. The results demonstrated that the concentrations of estrone and $17 \beta$-Estradiol were significantly higher in the high Gln level group than in the low Gln level group $(P<0.05$ and $P<0.001$, Figure $3 A-B)$. There were no statistically significant differences in the other hormone contents between the two groups ( $P>0.05$, Figure $3 C-I)$. These results revealed that $\mathrm{Gln}$ and $\mathrm{NE}$ in follicular fluid played important roles in promoting estrogen synthesis and the production. 
Oxidative metabolism is the major oxygen consumer in the follicle, which could be affected by amino acid metabolism. Antioxidants are indicated as factors that can maintain the balance between ROS reproduction and clearance. Then, we detected the expression levels of the antioxidant genes in GCs via qRT-PCR between the two groups. In the group of high GIn level, antioxidant gene IDH1 and glutathione peroxidase 1 (GPX1) increased significantly in GCs compared with that in group of low Gln level $(P<0.001$ and $\mathrm{P}<0.0001$, Figure 4A-B). Moreover, the expression of nuclear factor erythroid 2-related factor 2 (NRF2) and its endogenous inhibitor, kelch-like epichlorohydrin-associated protein-1 (KEAP1), significantly increased in the high Gln level group ( $P<0.01$, Figure 4C-D). Notably, the expression level of IDH1 mRNA in GCs showed a positive correlation with the GIn concentration in follicular fluid $(R=0.3822, P<0.05$, Figure $4 \mathrm{E})$. Furthermore, there was a positive correlation between the NE concentration in follicular fluid and IDH1 expression in GCs $(R=0.4009, P<0.05$, Figure 4F). To further clarify whether this difference in the expression of antioxidative genes was caused by individual differences, we summarized the clinical data of the IVF patients as Table 3 and found that there were no statistically significant differences in age $(P=0.1601)$, body mass index ( $B M I, P=0.5927)$, serum Anti-Mullerian Hormone ( $A M H, P=0.6033$ ) or thyrotropin (TSH, $\mathrm{P}=0.8698)$ between this two groups. These results demonstrated that the contents of GIn and NE in follicular fluid might affect the antioxidant capacity of GCs. 
Table 3

Characteristics of patients assessed for follicular fluid analysis

\begin{tabular}{|llll|}
\hline Characteristics & High level of Glutamine & Low level of Glutamine & P-value \\
\hline & $(\mathrm{n}=14)$ & $(\mathrm{n}=13)$ & \\
\hline Age(years) & $31.00[28.75-36.00]$ & $31.00[26.50-33.00]$ & 0.1601 \\
\hline BMI(Kg/m2) & $21.16[19.52-23.00]$ & $19.95[18.31-21.52]$ & 0.5927 \\
\hline Duration of infertility & $2.50[1.00-4.63]$ & $1.00[1.00-3.00]$ & 0.0472 \\
\hline Previous pregnancy & $2.00[1.00-3.00]$ & $0[0-1.00]$ & 0.0164 \\
\hline Previous delivery & $0[0-0.25]$ & $0[0-0]$ & 0.0820 \\
\hline AMH(ng/ml) & $2.35[1.29-4.29]$ & $2.90[1.93-3.81]$ & 0.6033 \\
\hline TSH(mIU/L) & $1.91[1.45-3.03]$ & $2.18[1.83-2.70]$ & 0.8698 \\
\hline Antral follicle count & $9.50[5.75-12.25]$ & $9.00[8.50-12.50]$ & 0.9911 \\
\hline Indication for IVF & & & 0.3289 \\
\hline Idiopathic & $1(7.14 \%)$ & $1(7.69 \%)$ & \\
\hline Male factor & $1(7.14 \%)$ & $0(0)$ & \\
\hline Endometriosis & $4(28.58 \%)$ & $2(15.38 \%)$ & \\
\hline Tube factor & $6(42.86 \%)$ & $4(46.15 \%)$ & \\
\hline Multiple & $2(14.28 \%)$ & $4(30.77 \%)$ & \\
\hline Data are median (interquartile range (IQR)) or numeric value (\%) & & \\
\hline AMH: anti-Mullerian hormone & & \\
\hline
\end{tabular}

IDH1 expression in granulosa cells is related with the quality of embryo development of IVF patients.

To determine whether follicular development and oocyte growth were different between the high Gln level and low GIn level groups, we compared several clinical indices in the two groups, including the number of mature follicles (diameters $\geq 14 \mathrm{~mm}$ ) on the human chorionic gonadotrophin (HCG) injection day, the number of retrieved oocytes, the fertilization rate, and the number and percentage of higher-quality embryos. There were no significant differences between the two groups in the above parameters $(P>0.05$, Figure 5A). Pearson correlation analysis was used to identify the relationship between the Gln/NE concentration in the follicular fluid and the ratio of higher-quality/cleaved embryos, and IDH1 expression in granulosa cells and the ratio of higher-quality/cleaved embryos. Results showed that there were no significant correlation between the GIn/NE concentration in the follicular fluid and the ratio of higherquality/cleaved embryos ( $R=0.1158$ and $R=0.0134, P>0.05$, Figure 5B-C). However, a significantly positive correlation was observed between IDH1 gene expression in GCs and the ratio of higher-quality/cleaved 
embryos ( $R=0.4766, P<0.05$, Figure $5 D)$. These results demonstrated that the IDH1 expression in cumulus GCs is directly related with the quality of oocyte and embryo development in IVF patients.

\section{Glutamine/norepinephrine improve the function of granulosa cells in vitro}

Previous studies have reported that GIn is required for oocyte maturation and embryo development [32] and adrenergic activity through NE participates in the control of follicular development and steroidal secretion [33]. To study the roles of GIn and NE in granulosa cells, the proliferation of cultured KGN cells in vitro was evaluated via EdU staining and western blotting. Fluorescent staining showed that the percentage of EdU-positive cells was dramatically reduced in the GIn deprivation group. The addition of NE partially increased the ratio of EdU-positive KGN cells. Importantly, GIn supplementation increased significantly the EdU-positive number ( $\mathrm{P}<0.0001$, Figure 6A-B). Moreover, the concentrations of E2 and Prog in the cell culture supernatant were measured by ELISA. The results showed that the concentration of E2 was reduced in the GIn deprivation group, and adding GIn to the medium significantly increased E2 production in KGN cells, which could be further improved by NE supplementation ( $P<0.01$, Figure $6 C$ ). Although GIn deprivation reduced the level of Prog in the cell supernatant, GIn and NE supplementation had no obvious effect on Prog production in KGN cells ( $(P<0.05$, Figure 6D). The expression of PCNA protein in KGN cells exhibited similar trends to the morphological results in the different treatment groups. Notably, GIn deprivation obviously reduced IDH1 protein expression in GCs, which could be recovered by NE and $G$ In supplementation ( $P<0.0001, P<0.01$ and $P<0.05$, Figure $6 E-G)$. These results revealed that the neurotransmitters GIn and NE could synergistically enhance the cell proliferation of granulosa cells and increase the E2 production.

\section{Discussion}

Follicular fluid is an important external environment for the oocyte growth and follicular development. Ideally, follicular fluid, a superfluous, abundant and easily available biological sample in IVF cycles, would be the optimum source of noninvasive prediction of oocyte quality [34]. Currently, investigations of follicular fluid biomarkers for oocyte quality mainly focus on measuring steroid hormones secreted by granulosa cells, such as estradiol and progesterone. Some studies have demonstrated that high levels of Prog and E2 in follicular fluid are positively related to the quality of oocyte and could be indicators for assessing oocyte mature and development. However, other studies have reported no correlation between hormones in the follicular fluid and oocyte quality or that they may even be correlated with a lower quality [35]. Steroid hormone production mainly relies on the function of granulosa cells, which are dynamically regulated by small molecule metabolites in follicular fluid. Therefore, exploring the relationship between metabolites and granulosa cell function may be conducive to search reliable biomarkers for assessing oocyte quality and predicting the outcome of IVF patients.

A metabolic approach is a powerful tool to analyze the complexities of follicular fluid. Previous study reported the application of gas chromatography-mass spectrometry (GC-MS) to detect polybrominated diphenyl ethers (PBDEs) in follicular fluid and found that the presence of PBDEs could be associated with 
failed embryo implantation[36]. In the current study, a more simple, fast and efficient LC-MS/MS technology was successfully applied for determination of simultaneous neurotransmitter in human follicular fluid. Consistent with other studies, we found that many different neurotransmitter metabolites exist in human follicular fluid, including Ach, GIn, Glu, His, NE, Tyr, Trp and Kyn, as shown in Table 2. Among them, high levels of GIn and NE have attracted our attention; there was a positive relationship between the contents of GIn and NE in follicular fluid. NE is released from ovarian nerve terminals wherein the ovarian compartment is directly innervated by sympathetic nerves [33]. Whether ovarian sympathetic nerves control the concentration of GIn in follicular fluid needs to be further studied.

Currently, extensive scientific attention has been given to an important metabolite Gln, which is one of the most abundant amino acid in the human body, such as skeletal muscles, plasma [37] and follicular fluid [38]. It has anti-inflammatory and antioxidant functions as well as effects that modulate the heat-shock protein response during stress; moreover, as a precursor of glutamate, GIn participates in multiple neurotransmitter metabolic pathways $[37,39,40]$. In addition, peripheral neurotransmitter NE participates in the control of steroidal secretion from the ovary and follicular development [33]. To study the effect of intra-follicular GIn and NE on cumulus granulosa cell function, we compared the expression of proliferation- and steroid hormone synthesis-related genes between the low Gln level and high GIn level groups. The results showed that GIn and NE could promote granulosa cell proliferation and steroid hormone synthase gene expression. To confirm this relationship, hormone synthetic metabolites were also quantitatively analyzed by LC-MS/MS technology. Among the many hormones, only the concentrations of estriol and $17 \beta$-estradiol were significantly decreased in the low Gln level group. These findings indicated that the content of GIn and NE in follicular fluid plays important roles in maintaining the proliferation of cumulus GCs and E2 production.

An altered follicular microenvironment may directly damage follicle recruitment and growth. Previous studies show that metabolites in the follicular fluid are direct or indirect regulators of oxidative stress and the function of granulosa cells [41]. A research found that Gln was used as an additive substance to enhance antioxidant capacity in the breast muscle of heat-stressed broilers [42]. Moreover, GIn improves the body's resistance to stress [43] and restores the ovarian function of PCOS rats [44]. In addition, GIn, as the main energy supply substance for mitochondria to form ATP, is an important source of energy for oocyte maturation and the development of embryo [38]. Therefore, Gln has become an essential component in embryo culture medium. A study reported that human ovarian tissue was cryopreserved using freezing medium supplemented with cryoprotectants and antioxidants (L-glutamine and taurine) and exhibited good preservation of ovarian vascular integrity and functionality of granulosa cells post thawing [45]. Furthermore, the glutamine supplementation could effectively alleviate inflammatory reaction and oxidative stress in PCOS rats [44] and protect Chinese hamsters from radiation damage [46]. Thus, the relationships among the levels of GIn in follicular fluid, cumulus GC function, and oocyte development are worthy of further study.

Excessive ROS damages DNA, reduces cell proliferation, and induces mitochondrial-mediated apoptosis in GCs, then affecting oocyte development $[47,48]$. Studies have demonstrated that there is a correlation 
between the apoptosis of GCs and poor oocyte quality and IVF outcomes in patients with PCOS [11, 49]. Many environmental stressors may cause inferior oocyte competence and alter oocyte development and growth by affecting GC function [48, 50,51]. The bidirectional interplay between cells and microenvironment affects the expression of genes and ultimately alter cellular behaviors by dynamic reciprocity [52]. In the current study, we found that GIn and NE not only upregulated IDH1 gene expression but also increased GPX1 expression in cumulus GCs. Notably, the concentration of GIn and NE in the follicular fluid were positively related to IDH1 gene expression in cumulus GCs, which could be a predictor of high-quality embryos. To date, a clear correspondence between specific metabolites in follicular fluid and measurable embryo-related variables has not been established. Taking the level of Gln and NE in follicular fluid and antioxidant gene expression in cumulus granulosa cells into account will provide more accurate information to help embryologists choose optimal quality oocytes.

There are several limitations in current study. First, the number of clinical samples was insufficient. Although the number of samples in the two groups met the statistical requirements, the limited number of samples influenced the subsequent analysis of reproductive outcomes of IVF patients. The second limitation was that only antioxidant genes expressions were assayed in cumulus GCs, however, other oxidative stress makers including ROS, MDA, GSH did not detected due to lack of enough samples. Finally, only KGN cells were used to confirm the roles of GIn and NE in maintaining granulosa cell function in vitro experiments. Whether appropriate supplementation of Gln and/or NE in animal models with ovarian dysfunction could improve fertility outcomes needs to be explored in future studies.

\section{Conclusions}

In summary, our research demonstrates that the concentrations of GIn and NE in follicular fluid influences proliferation and steroidogenesis in human cumulus GCs. Moreover, intrafollicular Gln and NE could upregulate the expression of the antioxidative gene IDH1 in cumulus GCs, which could be a good predictor for evaluating the quality of embryonic development.

\section{Abbreviations}

5-HIAA: 5-Hydroxyindole-3-acetic acid; 5-HT: Serotonin hydrochloride; 5-HTTP: 5-Hydroxytryptophan; Ach: Acetylcholine chloride; Ala: alanine; ART: Assisted reproductive technology; AMH: Anti-Mullerian Hormone; BMI: body mass index; CCND2: cyclin D2; DA: Hydroxytyramine hydrochloride; DOPA: Levodopa; E: Adrenaline hydrochloride; E2: 17b-estradiol; FSHR囚follicle stimulating hormone receptor; GABA: 4Aminobutyric acid; GCs: granulosa cells; GC-MS: gas chromatography-mass spectrometry; GPX1: glutathione peroxidase 1; GIn: Glutamine; Glu: Glutamate; Gly: Glycine; His: L-Histidine; HisA: Histamine; HCG: human chorionic gonadotrophin; HPLC: high-performance liquid chromatography; HPLC-MS/MS: high-performance liquid chromatography-mass spectrometry; HSD-3b: hydroxysteroid dehydrogenase3beta; HSD-17b: hydroxysteroid dehydrogenase-17beta; ICSI: intracytoplasmic sperm injection; IDH1: isocitrate dehydrogenase 1; IPMCH: International Peace Maternity and Child Health Hospital; IVF: in vitro fertilization; IVF-ET: in vitro fertilization and embryo transfer; KEAP1: kelch-like epichlorohydrin-associated 
protein-1; Kyn: Kynurenine; KynA: Kynurenic acid; LC-MS/MS: liquid chromatography-tandem mass spectrometry; LHR: luteinizing hormone receptor; LOD: limit of detection; LOQ: limit of quantification; MRM: multiple reaction monitoring; MT: Melatonine; NE: norepinephrine; NRF2: nuclear factor erythroid 2related factor 2; OCC: oocyte cumulus complex; PA: Picolinic acid; PBDEs: polybrominated diphenyl ethers; PCNA: proliferating cell nuclear antigen; PCOS: polycystic ovary syndrome; Pro: Proline; Prog: progesterone; ROS: reactive oxygen species; STAR: steroid acute regulatory protein; Trp: Tryptophan; TrpA: Tryptamine; TSH: thyrotropin; Tyr: Tyrosine; TyrA: Tyramine; XA: Xanthurenic acid; WHO: World Health Organization; VWA: Vanillymandelic Acid;

\section{Declarations}

\section{Consent for publication}

All authors have agreed to publish this article.

\section{Availability of data and materials}

The datasets generated and/or analysed during the current study are available in the the ProteomeXchange Consortium (https://proteomecentral.proteomexchange.org) with the dataset identifier px-submission \#562857 and px-submission \#562860.

\section{Competing interests}

The authors report no declarations of interests.

\section{Funding}

This work was supported by the National Key Research and Developmental Program of China (2018YFC1004800 and 2018YFC1004802), the National Natural Science Foundation of China (81971334 and 81701397) and the Shanghai Municipal Council for Science and Technology (20JC1412100 and 20ZR1463200).

\section{Authors' contributions}

Study design: D.L. and Q.Z.; Data collection: Q.Z., L.W., C.Z. and J.S.; Data analysis and interpretations: Q.Z., L.W., C.Z. and J.S.; Drafting the manuscript: D.L., Q.Z. and L.W. All the above authors revised and approved the manuscript and take responsibility for the integrity of the data.

\section{Acknowledgements}

Not applicable.

\section{Authors' information}


${ }^{1}$ The International Peace Maternity and Child Health Hospital, School of Medicine, Shanghai Jiao Tong University; Shanghai Key Laboratory of Embryo Original Diseases; Shanghai, China.

\section{References}

1. Gurunath S, Pandian Z, Anderson RA, Bhattacharya S: Defining infertility-a systematic review of prevalence studies. Hum Reprod Update 2011, 17:575-588.

2. Farquhar CM, Bhattacharya S, Repping S, Mastenbroek S, Kamath MS, Marjoribanks J, Boivin J: Female subfertility. Nat Rev Dis Primers 2019, 5:7.

3. Boivin J, Bunting L, Collins JA, Nygren KG: International estimates of infertility prevalence and treatment-seeking: potential need and demand for infertility medical care. Hum Reprod 2007, 22:1506-1512.

4. Öztürk R, Herbell K, Morton J, Bloom T: "The worst time of my life": Treatment-related stress and unmet needs of women living with infertility. J Community Psychol 2021, 49:1121-1133.

5. Drakopoulos P, Bardhi E, Boudry L, Vaiarelli A, Makrigiannakis A, Esteves SC, Tournaye H, Blockeel C: Update on the management of poor ovarian response in IVF: the shift from Bologna criteria to the Poseidon concept. Ther Adv Reprod Health 2020, 14:2633494120941480.

6. Schmalbrock LJ, Weiss G, Rijntjes E, Reinschissler N, Sun Q, Schenk M, Schomburg L: Pronounced Trace Element Variation in Follicular Fluids of Subfertile Women Undergoing Assisted Reproduction. Nutrients 2021, 13:4134.

7. Yang R, Niu ZR, Chen LX, Liu P, Li R, Qiao J: Analysis of related factors affecting cumulative live birth rates of the first ovarian hyperstimulation in vitro fertilization or intracytoplasmic sperm injection cycle: a population-based study from 17,978 women in China. Chin Med J (Engl) 2021, 134:14051415.

8. Da Broi MG, Giorgi VSI, Wang F, Keefe DL, Albertini D, Navarro PA: Influence of follicular fluid and cumulus cells on oocyte quality: clinical implications. J Assist Reprod Genet 2018, 35:735-751.

9. Dumesic DA, Meldrum DR, Katz-Jaffe MG, Krisher RL, Schoolcraft WB: Oocyte environment: follicular fluid and cumulus cells are critical for oocyte health. Fertil Steril 2015, 103:303-316.

10. Komatsu K, Masubuchi S: Mouse oocytes connect with granulosa cells by fusing with cell membranes and form a large complex during follicle development. Biol Reprod 2018, 99:527-535.

11. Gong Y, Luo S, Fan P, Zhu H, Li Y, Huang W: Growth hormone activates PI3K/Akt signaling and inhibits ROS accumulation and apoptosis in granulosa cells of patients with polycystic ovary syndrome. Reprod Biol Endocrinol 2020, 18:121.

12. Khan A, Dou J, Wang Y, Jiang X, Khan MZ, Luo H, Usman T, Zhu H: Evaluation of heat stress effects on cellular and transcriptional adaptation of bovine granulosa cells. J Anim Sci Biotechnol 2020, 11:25.

13. D'Albora H, Anesetti G, Lombide P, Dees WL, Ojeda SR: Intrinsic neurons in the mammalian ovary. Microsc Res Tech 2002, 59:484-489. 
14. Ben-Jonathan N, Arbogast LA, Rhoades TA, Bahr JM: Norepinephrine in the rat ovary: ontogeny and de novo synthesis. Endocrinology 1984, 115:1426-1431.

15. Barria A, Leyton V, Ojeda SR, Lara HE: Ovarian steroidal response to gonadotropins and betaadrenergic stimulation is enhanced in polycystic ovary syndrome: role of sympathetic innervation. Endocrinology 1993, 133:2696-2703.

16. Saller S, Merz-Lange J, Raffael S, Hecht S, Pavlik R, Thaler C, Berg D, Berg U, Kunz L, Mayerhofer A: Norepinephrine, active norepinephrine transporter, and norepinephrine-metabolism are involved in the generation of reactive oxygen species in human ovarian granulosa cells. Endocrinology 2012, 153:1472-1483.

17. Bedard K, Krause KH: The NOX family of ROS-generating NADPH oxidases: physiology and pathophysiology. Physiol Rev 2007, 87:245-313.

18. Hong J, Lee E: Intrafollicular amino acid concentration and the effect of amino acids in a defined maturation medium on porcine oocyte maturation, fertilization, and preimplantation development. Theriogenology 2007, 68:728-735.

19. Zhang CM, Zhao Y, Li R, Yu Y, Yan LY, Li L, Liu NN, Liu P, Qiao J: Metabolic heterogeneity of follicular amino acids in polycystic ovary syndrome is affected by obesity and related to pregnancy outcome. BMC Pregnancy Childbirth 2014, 14:11.

20. Andersen JV, Christensen SK, Westi EW, Diaz-delCastillo M, Tanila H, Schousboe A, Aldana BI, Waagepetersen HS: Deficient astrocyte metabolism impairs glutamine synthesis and neurotransmitter homeostasis in a mouse model of Alzheimer's disease. Neurobiol Dis 2021, 148:105198.

21. Bak LK, Schousboe A, Waagepetersen HS: The glutamate/GABA-glutamine cycle: aspects of transport, neurotransmitter homeostasis and ammonia transfer. J Neurochem 2006, 98:641-653.

22. Hardy MLM, Day ML, Morris MB: Redox Regulation and Oxidative Stress in Mammalian Oocytes and Embryos Developed In Vivo and In Vitro. Int J Environ Res Public Health 2021, 18.

23. Lim J, Luderer U: Oxidative damage increases and antioxidant gene expression decreases with aging in the mouse ovary. Biol Reprod 2011, 84:775-782.

24. Sun J, Guo Y, Fan Y, Wang Q, Zhang Q, Lai D: Decreased expression of IDH1 by chronic unpredictable stress suppresses proliferation and accelerates senescence of granulosa cells through ROS activated MAPK signaling pathways. Free Radic Biol Med 2021, 169:122-136.

25. Tamura H, Jozaki M, Tanabe M, Shirafuta Y, Mihara Y, Shinagawa M, Tamura I, Maekawa R, Sato S, Taketani T, et al: Importance of Melatonin in Assisted Reproductive Technology and Ovarian Aging. Int J Mol Sci 2020, 21.

26. Truong T, Gardner DK: Antioxidants improve IVF outcome and subsequent embryo development in the mouse. Hum Reprod 2017, 32:2404-2413.

27. van den Ouweland JM, Kema IP: The role of liquid chromatography-tandem mass spectrometry in the clinical laboratory. J Chromatogr B Analyt Technol Biomed Life Sci 2012, 883-884:18-32. 
28. Wang LS, Zhang MD, Tao X, Zhou YF, Liu XM, Pan RL, Liao YH, Chang Q: LC-MS/MS-based quantification of tryptophan metabolites and neurotransmitters in the serum and brain of mice. $\mathrm{J}$ Chromatogr B Analyt Technol Biomed Life Sci 2019, 1112:24-32.

29. Xu H, Wang Z, Zhu L, Sui Z, Bi W, Liu R, Bi K, Li Q: Targeted Neurotransmitters Profiling Identifies Metabolic Signatures in Rat Brain by LC-MS/MS: Application in Insomnia, Depression and Alzheimer's Disease. Molecules (Basel, Switzerland) 2018, 23:2375.

30. Hlisníková H, Petrovičová I, Kolena B, Šidlovská M, Mlynček M: Effect of prenatal phthalate exposure on the association of maternal hormone levels during early pregnancy and reproductive markers in infants at the age of 3 months. Reprod Toxicol 2021, 102:35-42.

31. Li XH, Ma YG, Geng LH, Qin L, Hu H, Li SW: Baseline psychological stress and ovarian norepinephrine levels negatively affect the outcome of in vitro fertilisation. Gynecol Endocrinol 2011, 27:139-143.

32. Zuo Z, Niu Z, Liu Z, Ma J, Qu P, Qiao F, Su J, Zhang Y, Wang Y: The effects of glycine-glutamine dipeptide replaced I-glutamine on bovine parthenogenetic and IVF embryo development. Theriogenology 2020, 141:82-90.

33. Garrido MP, Fernandois D, Venegas M, Paredes AH: Effects of sympathectomy on ovarian follicular development and steroid secretion. Reproduction 2018, 155:173-181.

34. Mendoza C, Ruiz-Requena E, Ortega E, Cremades N, Martinez F, Bernabeu R, Greco E, Tesarik J: Follicular fluid markers of oocyte developmental potential. Hum Reprod 2002, 17:1017-1022.

35. McRae C, Sharma V, Fisher J: Metabolite Profiling in the Pursuit of Biomarkers for IVF Outcome: The Case for Metabolomics Studies. Int J Reprod Med 2013, 2013:603167.

36. Johnson PI, Altshul L, Cramer DW, Missmer SA, Hauser R, Meeker JD: Serum and follicular fluid concentrations of polybrominated diphenyl ethers and in-vitro fertilization outcome. Environ Int 2012, 45:9-14.

37. Nemati A, Alipanah-Moghadam R, Molazadeh L, Naghizadeh Baghi A: The Effect of Glutamine Supplementation on Oxidative Stress and Matrix Metalloproteinase 2 and 9 After Exhaustive Exercise. Drug Des Devel Ther 2019, 13:4215-4223.

38. Pocate-Cheriet K, Santulli P, Kateb F, Bourdon M, Maignien C, Batteux F, Chouzenoux S, Patrat C, Wolf JP, Bertho G, Chapron C: The follicular fluid metabolome differs according to the endometriosis phenotype. Reprod Biomed Online 2020, 41:1023-1037.

39. Shanware NP, Mullen AR, DeBerardinis RJ, Abraham RT: Glutamine: pleiotropic roles in tumor growth and stress resistance. J Mol Med (Berl) 2011, 89:229-236.

40. Raizel R, Leite JS, Hypólito TM, Coqueiro AY, Newsholme P, Cruzat VF, Tirapegui J: Determination of the anti-inflammatory and cytoprotective effects of l-glutamine and l-alanine, or dipeptide, supplementation in rats submitted to resistance exercise. Br J Nutr 2016, 116:470-479.

41. Yuan XQ, Du YY, Liu C, Guo N, Teng XM, Hua X, Yao YC, Deng YL, Zeng Q, Deng TR, Li YF: Phthalate metabolites and biomarkers of oxidative stress in the follicular fluid of women undergoing in vitro fertilization. Sci Total Environ 2020, 738:139834. 
42. Hu H, Chen L, Dai S, Li J, Bai X: Effect of Glutamine on Antioxidant Capacity and Lipid Peroxidation in the Breast Muscle of Heat-stressed Broilers via Antioxidant Genes and HSP70 Pathway. Animals (Basel) 2020, 10.

43. Zhao Y, Wang Q, Wang Y, Li J, Lu G, Liu Z: Glutamine protects against oxidative stress injury through inhibiting the activation of PI3K/Akt signaling pathway in parkinsonian cell model. Environ Health Prev Med 2019, 24:4.

44. Wu G, Hu X, Ding J, Yang J: The effect of glutamine on Dehydroepiandrosterone-induced polycystic ovary syndrome rats. Journal of ovarian research 2020, 13:57-57.

45. Sanfilippo S, Canis M, Romero S, Sion B, Dechelotte P, Pouly JL, Janny L, Smitz J, Brugnon F: Quality and functionality of human ovarian tissue after cryopreservation using an original slow freezing procedure. J Assist Reprod Genet 2013, 30:25-34.

46. Winters R, Matthews R, Ercal N, Krishnan K: Glutamine protects Chinese hamster ovary cells from radiation killing. Life Sci 1994, 55:713-720.

47. Hyatt HW, Zhang Y, Hood WR, Kavazis AN: Changes in Metabolism, Mitochondrial Function, and Oxidative Stress Between Female Rats Under Nonreproductive and 3 Reproductive Conditions. Reprod Sci 2019, 26:114-127.

48. Abdelnour SA, Swelum AA, Abd El-Hack ME, Khafaga AF, Taha AE, Abdo M: Cellular and functional adaptation to thermal stress in ovarian granulosa cells in mammals. J Therm Biol 2020, 92:102688.

49. Lai Q, Xiang W, Li Q, Zhang H, Li Y, Zhu G, Xiong C, Jin L: Oxidative stress in granulosa cells contributes to poor oocyte quality and IVF-ET outcomes in women with polycystic ovary syndrome. Front Med 2018, 12:518-524.

50. Khadrawy O, Gebremedhn S, Salilew-Wondim D, Taqi MO, Neuhoff C, Tholen E, Hoelker M, Schellander K, Tesfaye D: Endogenous and Exogenous Modulation of Nrf2 Mediated Oxidative Stress Response in Bovine Granulosa Cells: Potential Implication for Ovarian Function. Int J Mol Sci 2019, 20.

51. Ai A, Tang Z, Liu Y, Yu S, Li B, Huang H, Wang X, Cao Y, Zhang W: Characterization and identification of human immortalized granulosa cells derived from ovarian follicular fluid. Exp Ther Med 2019, 18:2167-2177.

52. Thorne JT, Segal TR, Chang S, Jorge S, Segars JH, Leppert PC: Dynamic reciprocity between cells and their microenvironment in reproduction. Biol Reprod 2015, 92:25.

\section{Figures}

Figure 1

Legend not included with this version. 
Figure 2

Legend not included with this version.

Figure 3

Legend not included with this version.

\section{Figure 4}

Legend not included with this version.

\section{Figure 5}

Legend not included with this version.

\section{Figure 6}

Legend not included with this version. 